\title{
Experimentar empatía en estudiantes de medicina: deprivación sensorial
}

\author{
Teresa Guilera, Iolanda Batalla, Jorge Soler-González
}

Objetivo. Identificar las emociones que experimentan los estudiantes de medicina en el contexto de deprivación sensorial
para mejorar la empatía.

Sujetos y métodos. Se presenta el programa del taller (deprivación visual, auditiva y discapacidad física). Se identifican y se miden las emociones mediante la escala Self-Assessment Manikin, y se mide la empatía médica mediante la escala de empatía médica de Jefferson.

Resultados y conclusiones. La deprivación sensorial permite experimentar diferentes tipos de emociones. Identificarlas permite al estudiante de medicina ser más sensible a las personas con discapacidad y aumentar la empatía.

Palabras clave. Educación médica. Emociones. Empatía. Estudiantes de medicina.

\section{Experiencing empathy in medical students: sensorial deprivation}

Aim. To identify emotions experienced by medical students in a workshop of sensory deprivation to enhance empathy.

Subjects and methods. The workshop program (visual deprivation, auditory deprivation and physical disabilities) is presented. Emotions are identified and measured by Self-Assessment Manikin scale. Medical empathy is measured by Jefferson Scale of Physician Empathy.

Results and conclusions. Sensory deprivation can experience different types of emotions. Identify them allows medical students to be more sensitive to people with disabilities and increase empathy.

Key words. Emotions. Empathy. Medical education. Medical students.

\section{Introducción}

La empatía médica es una habilidad eminentemente cognitiva que implica la comprensión de las experiencias, inquietudes y perspectivas del paciente, combinadas con la capacidad de comunicar esta comprensión y con la intención de ayudar. Una buena relación médico-paciente es crucial en la práctica médica y en el arte de curar. En la medida en que el médico entienda lo que el paciente piensa y siente, mejor será la atención que pueda ofrecer [1].

En el contexto de la empatía médica, un estudio de Hojat et al propone el concepto de orientación empática frente a orientación simpática. Define la simpatía como el resultado de un proceso pasivo, la capacidad de compartir afecto entre individuos, experimentando una emoción más o menos similar ('sentir'), y define la empatía como el resultado de un proceso activo en el que compartir las emociones lleva a un mejor entendimiento del sujeto observado ('entender'). Hojat et al [2] proponen que la compasión reside en el área en el que se superpo- nen la empatía y la simpatía, donde ambos atributos se expresan en cantidades moderadas y en las que la cognición y la emoción interactúan para producir la respuesta empática genuina.

Diversos autores proponen diferentes estrategias para mejorar la empatía en la formación médica. Una revisión sistemática realizada entre 2004 y 2012 identificó 18 artículos que describían intervenciones educativas diseñadas para desarrollar la empatía en estudiantes de medicina, 15 de los cuales mostraban un aumento significativo de la empatía tras dichas intervenciones. En otros estudios se ha evidenciado un aumento de empatía, aunque volviendo a niveles previos a las pocas semanas del taller $[3,4]$.

Un estudio piloto de la Universidad de Jefferson experimentó en médicos residentes el 'shadowing patients': durante unas horas, el residente acompaña al paciente en el servicio de urgencias. Los autores sugieren que el desgaste de la empatía podría prevenirse con este tipo de experiencias [5].

No hemos encontrado en la bibliografía experimentación en deprivación sensorial en estudiantes
Servicio de Psiquiatría; Hospital Universitari de Santa Maria; Gestió de Serveis Sanitaris (T. Guilera, I. Batalla). Facultad de Medicina; Universitat de Lleida (I. Batalla, J. Soler-González). IRBLLEIDA (T. Guilera, I. Batalla, J. SolerGonzález). Lleida, España.

Correspondencia:

Dra. Teresa Guilera Lladós. Servicio de Psiquiatría. Hospital Universitari de Santa Maria. Gestió de Serveis Sanitaris. Avda. Rovira Roure, 44 E-25198 Lleida.

E-mail:

tguilera@gss.scs.es

Recibido:

01.09.16.

Aceptado:

13.09.16.

Conflicto de intereses:

No declarado.

Competing interests: None declared.

(c) 2017 FEM 
Tabla I. Programa del taller de sensibilización a la discapacidad.

Parte teórica (1 hora)

1. Introducción al concepto de empatía y su aplicación en la práctica médica

2. Información sobre el estudio, pautas para llevar a cabo la deprivación sensorial, consentimiento informado

3. Cuestionario inicial:

a) Escala de empatía médica de Jefferson (JSPE) de Hojat

b) Casos clínicos para medir la orientación empática y orientación simpática de Hojat

Parte práctica (1 hora): trabajo por parejas

1. Deprivación sensorial visual: circuito de 10 minutos de duración. Durante cinco minutos uno de los estudiantes se pone en el papel de ciego (antifaz) y después se cambian los papeles y se pone en el papel de guía del ciego

2. Deprivación sensorial auditiva: circuito de 10 minutos de duración. Durante cinco minutos uno de los estudiantes se pone en el papel de sordo (tapones desechables de oído) y después se cambian los papeles y se pone en el papel de guía del sordo

3. Discapacidad física: circuito de 10 minutos de duración (cinco minutos con silla de ruedas y cinco minutos como guía del compañero en silla de ruedas)

\section{Puesta en común}

1. Cuestionario final:

a) Escala Self-Assessment Manikin (SAM). Valoración de la emoción en su escala bidimensional: valencia (emoción positiva/negativa) y arousal (nivel de activación o intensidad de la emoción)

b) Identificación de las emociones experimentadas: miedo, alegría, tristeza, rabia y amor (en el que cognición y emoción interactúan para producir la respuesta empática genuina) como ciego, sordo, disminución física, la confianza en el guía, así como las emociones como guía.

c) Escala de empatía médica de Jefferson (JSPE) de Hojat.

2. Grupal: puesta en común de las experiencias vividas durante la parte práctica.

de medicina, pero teniendo en cuenta que es uno de los métodos para mejorar la empatía y, en concreto, la toma de perspectiva, el objetivo de este estudio piloto es la sensibilización del grupo sobre los problemas a los que se enfrentan las personas con discapacidad e identificar las emociones que se experimentan.

\section{Sujetos y métodos}

Este proyecto cuenta con la aprobación del Comité Ético de Investigación Clínica de Lleida, CEIC-1515. La población a estudio la forman 14 estudiantes de medicina de la Facultad de Medicina de la Universitat de Lleida, que mediante consentimiento informado aceptan participar de forma voluntaria en el taller de dos horas de duración. Una submuestra de cuatro estudiantes de medicina realizó también la deprivación auditiva y física, así como la valoración de la empatía al final del taller.
La tabla I muestra el programa del taller y los cuestionarios administrados:

- Escala de empatía médica de Jefferson -Jefferson Scale of Physician Empathy (JSPE) - de Hojat, que mide la empatía en el contexto de la educación médica y la atención al paciente [1].

- Casos clínicos para medir la orientación empática y la orientación simpática de Hojat [2].

- Escala Self-Assessment Manikin (SAM), en la que del 1 al 9 se valora la emoción en su escala bidimensional: valencia (emoción positiva/negativa) y arousal (nivel de activación o intensidad de la emoción) [6].

\section{Resultados}

La muestra de estudiantes de medicina está formada por un $78,6 \%$ de mujeres y un $21,4 \%$ de varones. El 78,6\% cursan quinto de medicina; el 14,3\%, cuarto, y el 7,1\%, tercero. La media de edad de los participantes es de 22,29 \pm 1,07 años.

La media de la empatía médica medida con la JSPE es de 120,79 $\pm 13,12$. En los casos clínicos de Hojat, la media de orientación empática (4-16) es de $13,50 \pm 2,1$, y la de orientación simpática (4-16), de $10,79 \pm 3,3$. Un $78,6 \%$ de los estudiantes tienen un predominio de orientación empática; un 14,3\%, orientación simpática, y un 7,1\% puntúan igual en ambas.

La tabla II muestra las emociones experimentadas en la deprivación sensorial y su medida en la escala bidimensional valencia/arousal. La deprivación visual es la que genera más malestar y la deprivación auditiva resulta la más desagradable. En la deprivación visual predomina el miedo en el papel de ciego y amor (respuesta empática genuina) como guía, y en la deprivación auditiva, la tristeza, tanto en el papel de sordo como en el de guía. En la discapacidad física predomina la rabia y, como guía, la tristeza.

En la submuestra de cuatro estudiantes, la media de la empatía médica medida con la JSPE, al inicio del taller, es de 109,50 $\pm 18,52$, y al final, de 116,50 \pm 22,55 , mostrando un aumento en la puntuación de la empatía en todos ellos.

\section{Discusión}

La deprivación sensorial permite experimentar diferentes tipos de emociones. Identificarlas y medirlas permite al estudiante de medicina ser más sensible a las personas con alguna discapacidad, ponerse en la piel del discapacitado/paciente y aprender a 
Tabla II. Emociones experimentadas en la deprivación sensorial.

\begin{tabular}{|c|c|c|c|c|c|c|c|}
\hline & & \multicolumn{2}{|c|}{ Deprivación visual $(n=14)$} & \multicolumn{2}{|c|}{ Deprivación auditiva $(n=4)$} & \multicolumn{2}{|c|}{ Disminución física $(n=4)$} \\
\hline & & Media & $\mathrm{DE}$ & Media & $\mathrm{DE}$ & Media & $\mathrm{DE}$ \\
\hline \multicolumn{2}{|c|}{ Valencia (1-9) (agradable/desagradable) } & 5,14 & 2,77 & 6,50 & 1,00 & 3,25 & 2,60 \\
\hline \multirow{2}{*}{\multicolumn{2}{|c|}{ Arousal (1-9) (calma/excitación) }} & 5,67 & 2,18 & 4,00 & 1,83 & 4,00 & 2,16 \\
\hline & & Ciego & Guía & Sordo & Guía & Silla de ruedas & Guía \\
\hline \multirow{6}{*}{$\begin{array}{l}\text { Emociones } \\
\text { experimentadas }\end{array}$} & Miedo & $71,4 \%$ & - & - & - & - & - \\
\hline & Alegría & $14,3 \%$ & $35,7 \%$ & - & - & - & - \\
\hline & Tristeza & $14,3 \%$ & $14,3 \%$ & $50 \%$ & $25 \%$ & $25 \%$ & $25 \%$ \\
\hline & Rabia & - & - & $25 \%$ & $25 \%$ & $50 \%$ & $25 \%$ \\
\hline & Amor & - & $50 \%$ & $25 \%$ & $50 \%$ & $25 \%$ & $50 \%$ \\
\hline & Confianza en el guía & $100 \%$ & & $100 \%$ & & $100 \%$ & \\
\hline
\end{tabular}

DE: desviación estándar.

responder de forma adecuada como guía/médico con la intención de ayudar, objetivo principal del médico en su práctica clínica.

Teniendo en cuenta que estudios previos han demostrado que las intervenciones psicoeducativas pueden ser efectivas para mejorar la empatía en estudiantes de medicina [3], presentamos una propuesta de taller innovador, ya que trabaja con la experimentación directa de las emociones. Además, el trabajo en parejas y la posterior puesta en común con el grupo permite mejorar la actitud empática y conseguir, indirectamente, una mejor comprensión del paciente [1].

El número de participantes es una limitación del estudio. Nuestro grupo está trabajando en aumentar el tamaño muestral en las siguientes promociones. Conseguir la participación de los alumnos acostumbra a ser una tarea complicada, pero más allá del tamaño muestral, nos parece muy importante dar a conocer la metodología a la comunidad científica por ser un tema pionero de interés creciente en la docencia española.

Añadiremos también al programa del taller la experimentación en shadowing patients y, dados los resultados, la medida de la empatía médica (JSPE) antes y después del taller.

El análisis de los resultados obtenidos permitirá valorar la incorporación del taller en los estudios de pregrado de medicina.

\section{Bibliografía}

1. Alcorta A, González-Guerrero JF, Tavitas-Herrera SE, Rodríguez-Lara FJ, Hojat M. Validación de la escala de empatía médica de Jefferson en estudiantes de medicina mexicanos. Salud Mental 2005; 28: 57-63.

2. Hojat M, Spandorfer J, Louis DZ, Gonnella JS. Empathic and sympathetic orientations toward patient care: conceptualization, measurement, and psychometrics. Acad Med 2011; 86: 989-95.

3. Batt-Rawden SA, Chisolm MS, Anton B, Flickinger TE. Teaching empathy to medical students: an updated, systematic review. Acad Med 2013; 88: 1171-7.

4. Hojat M. Ten approaches for enhancing empathy in health and human services cultures. J Health Hum Serv Adm 2009; 31: 412-50.

5. Forstater AT, Chauhan N, Allen A, Hojat M, Lopez BL. An emergency department shadowing experience for emergency medicine residents: can it prevent the erosion of empathy? Acad Emerg Med 2011; 18: S2.

6. Bradley MM, Lang PJ. Measuring emotion: the self-assessment manikin and the semantic differential. J Behav Ther Exp Psychiatry 1994; 25: 49-59. 